\title{
Generation of Squeezing in Higher Order Hermite-Gaussian Modes with an Optical Parametric Amplifier
}

\author{
M. Lassen \\ mlassen@fysik.dtu.dk

\section{Delaubert} \\ C.C. Harb \\ P.K. Lam \\ N. Treps
}

H-A. Bachor

\begin{abstract}
The Australian National University, ACQOA, Canberra ACT 0200, Australia Department of Physics, DTU, Building 309, DK-2800 Lyngby, Denmark.

The Australian National University, ACQOA, Canberra ACT 0200, Australia Laboratoire Kastler Brossel, 4 place Jussieu, case 74, Paris 75252 Cedex 05, France.

The Australian National University, ACQOA, Canberra ACT 0200, Australia School of Information Technology and Electrical Engineering, University College, The University of New South Wales, Canberra, ACT, 2600.
\end{abstract}

The Australian National University, ACQOA, Canberra ACT 0200, Australia.

Laboratoire Kastler Brossel, 4 place Jussieu, case 74, Paris 75252 Cedex 05, France.

The Australian National University, ACQOA, Canberra ACT 0200, Australia.

We demonstrate quantum correlations in the transverse plane of continuous wave light beams by producing $-4.0 d B,-2.6 d B$ and $-1.5 \mathrm{~dB}$ of squeezing in the TEM $\mathrm{TE}_{00}, \mathrm{TEM}_{10}$ and $\mathrm{TEM}_{20}$ Hermite-Gauss modes with an optical parametric amplifier, respectively. This has potential applications in quantum information networking, enabling parallel quantum information processing. We describe the setup for the generation of squeezing and analyse the effects of various experimental issues such as mode overlap between pump and seed and nonlinear losses. [DOI: 10.2971/jeos.2006.06003]

Keywords: Imaging and optical processing, quantum optics, nonclassical field states, quantum fluctuations, quantum noise, and quantum jumps

\section{Introd uction}

There has been a growing interest during recent years in spatial quantum optical effects, usually called quantum imaging effects [1, 2, 3], as the generation of spatial correlations or spatial squeezing in the transverse plane of laser beams may open the way for new applications in many different areas. Among them are biophotonics, laser physics, astronomy and quantum information. Some pertinent examples are the measurement of small transverse displacement and tilt of a TEM 00 laser beam below the quantum noise limit [4, 5], detection of weak phase images [1], quantum teleportation of optical information [6], transverse spatial quantum correlation for transmission of images [3] and noiseless image amplification [7]. Multiple spatial modes can also provide advantages in regard to the complexity of quantum information protocols [8] and can allow parallel transfer of quantum information through an optical network. In single photon optics, this has created considerable interest in the use of modes with different angular momentum [9, 10, 11, 12]. An advantage of continuous wave light beams is that close to perfect modulation and detection schemes are available, which is a requirement for the effective use of squeezed and entangled light in quantum information protocols.

Several orthonormal basis are available to describe the spatial properties of laser beams. The most commonly used are the
Hermite-Gauss (H-G) and the Laguerre-Gauss basis [13]. In this contribution will we concentrate on the H-G modes, but a similar study could be undergone with another set of modes. The higher order H-G modes are particularly interesting with a cartesian description of the transverse plane, as they are directly related to simple spatial properties of Gaussian beams [14]. The real and imaginary parts of the $\mathrm{TEM}_{10}$ mode represent small changes in tilt and position of a TEM 00 beam [5], whereas the real and imaginary parts of the $\mathrm{TEM}_{20}$ mode correspond to a small waist-size and waist-position mismatch [15]. Using electro-optic spatial modulators, the information can be encoded directly into the H-G modes. The information can then be extracted using homodyne detections with adapted transverse profiles for the local oscillator [16, 5]. In this way we can encode and detect parallel quantum information in the transverse plane of continuous wave light beams. In order to apply quantum information protocols to the transverse plane of continuous wave light beams, what was missing until now was a reliable and efficient source for the generation of squeezing in different higher order $\mathrm{H}-\mathrm{G}$ modes. We propose here a method to fill this gap.

The present paper is devoted to the experimental study of squeezing in higher order $\mathrm{H}-\mathrm{G}$ modes using an optical parametric amplifier (OPA). In the past OPAs have proven to be 
efficient sources for the generation of quadrature squeezed light. Since the first generation of squeezed light by parametric down-conversion by $\mathrm{Wu}$ et al. in 1986 [17], squeezed noise power variances of up to $-7 \mathrm{~dB}[18]$, and squeezing at sideband frequencies down to sub-kHz frequencies [19] have been demonstrated with OPAs. However squeezing has so far been limited to $\mathrm{TEM}_{00}$ mode operation. We demonstrate here an experimental technique to produce squeezed light in different $\mathrm{H}-\mathrm{G}$ modes, namely the $\mathrm{TEM}_{10}$ and $\mathrm{TEM}_{20}$ modes, using an OPA. We report $-4.0 \mathrm{~dB},-2.6 \mathrm{~dB}$ and $-1.5 \mathrm{~dB}$ of observed squeezing for the TEM $00, \mathrm{TEM}_{10}$ and $\mathrm{TEM}_{20} \mathrm{H}-\mathrm{G}$ modes, respectively. The squeezing is generated in a back seeded OPA with a bulk lithium niobate $\left(\mathrm{MgO}: \mathrm{LiNbO}_{3}\right)$ type I nonlinear crystal, pumped by the second harmonic, from a continuous wave solid-state monolithic YAG laser.

The paper is organised as follows. In Section 2, we introduce a mode-overlap theory for second order nonlinear parametrical interaction with higher order H-G modes, in the thin crystal approximation. Section 3 describes our experimental setup and procedure to generate squeezing in different $\mathrm{H}-\mathrm{G}$ modes with an OPA. Section 4 addresses experimental issues such as the optimisation of the parametrical interaction. Section 5 presents our squeezing measurements. Finally, we conclude and present an outlook for further work in Section 6 .

\section{PTICAL PARAMETRIC AMPLI- FICATION AND IDEAL PUMP MODE FOR TE M no SQ UEEZIN G}

An OPA is a second order nonlinear optical device, where three optical beams are coupled parametrically to each other through the second order susceptibility $\chi^{(2)}$ of a nonlinear crystal, in an optical cavity. A pump photon of energy $\hbar \omega_{p}$ incident on an OPA down-converts into two photons, signal and idler, of energy $\hbar \omega_{s}$ and $\hbar \omega_{i}$ respectively, hence the name parametrical down-conversion [20]. In order to have a significant effect, the nonlinear interaction must satisfy energy $\left(\omega_{p}=\omega_{s}+\omega_{i}\right)$ and phase-matching $\left(k_{p}=k_{s}+k_{i}\right)$ conservation. In our experiment, the frequency of signal and idler are degenerate, i.e. $\omega_{s}=\omega_{i}$, and the polarisation of the pump is at $90^{\circ}$ to the polarisation of the signal and idler, corresponding to a type I phase-matching.

In order to pump the crystal efficiently for a $\mathrm{TEM}_{n 0}$ signal mode, the transverse profile of the pump mode must locally match with the square of the signal mode. We use $\left\{u_{i}\right\}$ as the notation for the fundamental signal and idler mode basis whose first mode has a waist of $w_{0}$, and $\left\{v_{i}\right\}$ for the second harmonic pump mode basis whose first mode has a waist of $w_{0} / \sqrt{2}$. In the thin crystal approximation and in the case of a $\mathrm{TEM}_{n 0}$ signal mode, the optimal pump profile $\mathcal{E}_{n}(\vec{r})$ is defined by:

$$
\mathcal{E}_{n}(\vec{r})=\sum_{i=1}^{n} \Gamma_{n i} v_{2 i}(\vec{r}) .
$$

where $\vec{r}$ is the transverse beam coordinate and $\Gamma_{n i}$ describes the spatial overlap between the squared signal and the second harmonic pump modes in the transverse plane, and is given by:

$$
\Gamma_{n i}=\int_{-\infty}^{\infty} \frac{u_{n}^{2}(\vec{r})}{\alpha_{n}} v_{2 i}(\vec{r}) d \vec{r},
$$

where $\alpha_{n}$ corresponds to the normalisation of the squared signal, and is defined by the relation $\alpha_{n}^{2}=\int_{-\infty}^{\infty} u_{n}^{4}(\vec{r}) d \vec{r}$. The optimal pump profile has only even components since the $\mathrm{TEM}_{n 0}$ signal squared profile is necessarily even, and its highest component is of order $2 n$. This decomposition is finite because the pre-exponential polynomial in the expression of $u_{n}^{2}$ is of order $2 n$, and therefore does not project onto higher order modes. The common case of using a TEM 00 signal mode yields $\Gamma_{00}=1$ and corresponds to a perfect spatial overlap as the optimal pump profile is also a TEM 00 mode. The presence of several non zero coefficients implies that for all cases, except a $\mathrm{TEM}_{00}$ pump mode, the optimal pump profile does not correspond to the signal intensity distribution. For a $\mathrm{TEM}_{10}$ signal mode resonant in the OPA cavity, the only non zero overlap coefficients calculated from Eq.(2) are given by:

$$
\begin{aligned}
& \Gamma_{10}=0.58 \\
& \Gamma_{12}=0.82 .
\end{aligned}
$$

As they are the only non zero ones, they fulfil $\Gamma_{10}^{2}+\Gamma_{12}^{2}=1$. For a $\mathrm{TEM}_{20}$ signal mode, the only pump modes which have non zero overlap in the cavity are the TEM $00, \mathrm{TEM}_{20}$ and $\mathrm{TEM}_{40}$ modes. The overlap coefficients are given by:

$$
\begin{aligned}
& \Gamma_{20}=0.47 \\
& \Gamma_{22}=0.44 \\
& \Gamma_{24}=0.77 .
\end{aligned}
$$

Again, they fulfil $\Gamma_{20}^{2}+\Gamma_{22}^{2}+\Gamma_{24}^{2}=1$. The presence of several non zero coefficients accounts for the multi-mode aspect of the ideal pump mode. Although generating such a complicated mode is in principle possible by using holograms [21] or forcing a laser cavity to emit in these mode [22, 23], we choose as a first step, to use a TEM 00 pump mode, as discussed in the next section.

Still using the thin crystal approximation with a multi-mode description of each field as in Ref. [24], we can show that pumping the OPA cavity with a non optimal mode results in an increase of the oscillation threshold. This increase is inversely proportional to the square of the overlap coefficient between the chosen pump mode and the optimal pump mode. For instance, when the signal mode resonant in the OPA is a $\mathrm{TEM}_{10}$ mode, pumping with a $\mathrm{TEM}_{00}$ mode increases the threshold by a factor $1 / \Gamma_{10}^{2} \simeq 3$. As for a $\mathrm{TEM}_{20}$ signal mode, pumping with a $\mathrm{TEM}_{00}$ mode increases the threshold by a factor $1 / \Gamma_{20}^{2} \simeq 4.5$. Since the threshold is directly related to the local intensity in the crystal, it will be further increased for higher order signal mode operations. Indeed, higher order mode intensity is more spread out in the transverse plane than for the $\mathrm{TEM}_{00}$ mode. Therefore the best conversion efficiency is expected to happen for a TEM 00 mode pump, independently from any overlap issue. Still in the thin crystal approximation, the threshold is further increased by a factor of 1.3 , and 1.6, respectively for $\mathrm{TEM}_{10}$ and $\mathrm{TEM}_{20}$ signal modes, relative to a $\mathrm{TEM}_{00}$ signal mode operation. The relative theoretical thresholds for the three first $\mathrm{H}-\mathrm{G}$ modes are presented in Table 1 in Section 4. 
We have estimated theoretically the threshold modifications imposed by the operation of an OPA with higher order transverse modes, and will use this knowledge in the next sections to interpret our experimental results.

\section{THE EXPERIM ENTAL SETUP}

The experimental squeezing setup is illustrated in Figure 1. To ensure stable squeezing, 6 locking loops are implemented in the experiment. For locking we use the Pound Drever Hall locking technique, where a phase-modulation is imparted on the optical beams with electric-optic modulators (EOM). The generated error-signals are then fed back to the cavities through piezo-electric elements (PZT) [25]. All cavities in the experiment are therefore held at resonance at the same time. The experiment stays locked for longer than 20 minutes. The only limiting factor is temperature fluctuations in the laboratory.

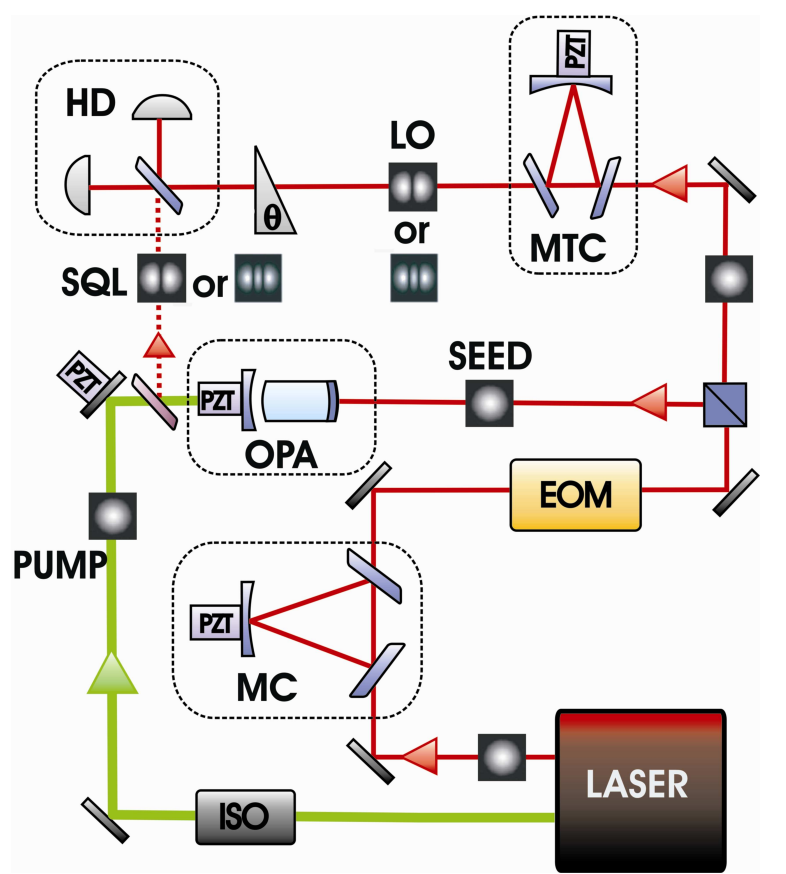

FIG. 1 Experimental setup to generate higher order transverse mode squeezing. An OPA is seeded with a misaligned $\mathrm{TEM}_{00}$ beam. The cavity is locked to the fundamental $\mathrm{TEM}_{n 0}$ mode and pumped with a second harmonic TEM 00 beam. The TEM $\mathrm{TE}_{n 0}$ squeezed beam is analysed using a homodyne detection (HD), whose $\operatorname{TEM}_{n 0}$ local oscillator is created from a misaligned ring cavity (MTC).

The experimental procedure for producing squeezing is as follows. The OPA is back seeded with a 1064 nm TEM 00 beam. The seed is misaligned into the OPA in order to excite higher order H-G modes. The OPA cavity is then locked to the TEM $n$ mode and pumped with a $\mathrm{TEM}_{00}$ second harmonic mode, wavelength $\lambda=532 \mathrm{~nm}$, generated internal in our cw solidstate monolithic YAG laser. The laser gives $950 \mathrm{~mW}$ of second harmonic power and $195 \mathrm{~mW}$ of infrared power [26]. The $1064 \mathrm{~nm}$ beam from the laser is first sent through a ring cavity, a so-called mode-cleaner (MC), which filters out the intensity and frequency noise of the laser above the bandwidth of the $\mathrm{MC}$. The MC also defines a high quality spatial mode. A bandwidth of $2.5 \mathrm{MHz}$ is measured and a transmission greater than
$90 \%$ is obtained for the $\mathrm{TEM}_{00}$ mode. The pump beam from the internal frequency doubler passes through an optical isolator (ISO: isolation $>40 \mathrm{~dB}$ ) and is carefully mode-matched (95\%) into the OPA. Working at pump powers below threshold, the seed is either amplified or de-amplified depending on the relative phase between the pump and the seed. This relative phase is stably locked to de-amplification in order to generate an amplitude quadrature squeezed beam.

We used a concentric hemilithic cavity design for the OPA, consisting of a $2 \times 2.5 \times 6.5 \mathrm{~mm}^{3}$ lithium niobate $\left(\mathrm{MgO}: \mathrm{LiNbO}_{3}\right)$ type I nonlinear crystal doped with $7 \%$ of magnesium. The birefringence of the crystal is highly temperature dependent; accurate temperature control of the crystal, typically at the milli-Kelvin level, is therefore required to achieve optimal phase-matching, as shown in Figure 2.

The back surface of the crystal is polished so that it has a $8 \mathrm{~mm}$ radius of curvature and is high reflectance coated for both wavelengths. The output coupler has $96 \%$ reflectivity for $1064 \mathrm{~nm}$ and 10\% reflectivity for $532 \mathrm{~nm}$, has a radius of curvature of $25 \mathrm{~mm}$, and is placed $23 \mathrm{~mm}$ from the front-end of the crystal. The cavity is therefore near concentricity with a waist of $24 \mu \mathrm{m}$ for the $1064 \mathrm{~nm}$ cavity and $19 \mu \mathrm{m}$ for the $532 \mathrm{~nm}$ cavity. The OPA has a finesse of approximately 165 with a free spectral range of $10 \mathrm{GHz}$ and a cold cavity bandwidth of $60 \mathrm{MHz}$. The TEM $\mathrm{T}_{n}$ squeezed beam generated at the output of the OPA and the pump beam are separated with dichroic mirrors. The squeezed beam is then analysed using a homodyne detection with a $\mathrm{TEM}_{n 0}$ local oscillator (LO), thus extracting only the information of the $\mathrm{TEM}_{n 0}$ component of the squeezed beam. The LO is created with a ring cavity, used as a mode transferring cavity (MTC) and designed to prevent any transverse mode degeneracy when locked to resonance on the $\mathrm{TEM}_{n 0}$ mode.

Before describing the measured squeezing we focus on the classical behaviour of the OPA.

\section{P TIM ISING THE PARAMET- RIC INTERACTION: GAIN AND THRESHOLD MEASUREMENTS}

In order to have large parametric interaction we need the best possible mode overlap between pump and seed modes. To achieve this, different issues have to be addressed such as mode-matching and alignment of pump and seed into the cavity. A useful tool for optimising the parametric interaction and at the same time the possible amount of squeezing to be extracted - is to measure the classical gain factor of the seed.

Since our OPA is not a cavity for the pump beam, the output coupler has only $10 \%$ reflectivity for $532 \mathrm{~nm}$, we can envision three different cases for pumping the OPA efficiently, knowing that we are restricted to a $\mathrm{TEM}_{00}$ pump mode operation, as discussed in the previous section. We can match the pump profile to the TEM 00 mode defined through the infrared mode; we can de-focus the pump; or, we can misalign the pump to match with one lobe (one side) of the infrared mode, in the $\mathrm{TEM}_{10}$ mode case for instance. In each case we 
have tuned the crystal temperature to maximise the gain and find that the most efficient option is to pump with a TEM 00 mode that is aligned with the cavity axis and optimally mode matched for maximum coupling to the cavity, as we would do for if we wanted to produce $\mathrm{TEM}_{00}$ squeezing. The disadvantage of this method was that the maximum pump power that could be delivered to the system was set by the threshold power of the $\mathrm{TEM}_{00}$.

The optimal temperature of the nonlinear crystal to produce squeezing differs for each $\mathrm{TEM}_{n 0}$, due to the different Gouy phase-shifts between H-G modes [27]. The temperature has thus to be re-optimised for each experiment. A direct manifestation of the shift in optimised phase-matching temperature is shown on Figure 2. This figure shows the classical gain factor measured as a function of the crystal temperature, for signal profiles given by the three first H-G modes, still using the best pumping option. We find that the optimal phasematching temperature are $62.1^{\circ} \mathrm{C}, 61.6^{\circ} \mathrm{C}$ and $60.6^{\circ} \mathrm{C}$ for the $\mathrm{TEM}_{00}, \mathrm{TEM}_{10}$ and $\mathrm{TEM}_{20}$ modes, respectively. The width (FWHM) for optimal phase-matching temperature is approximately $1^{\circ} \mathrm{C}$ for all three cases.

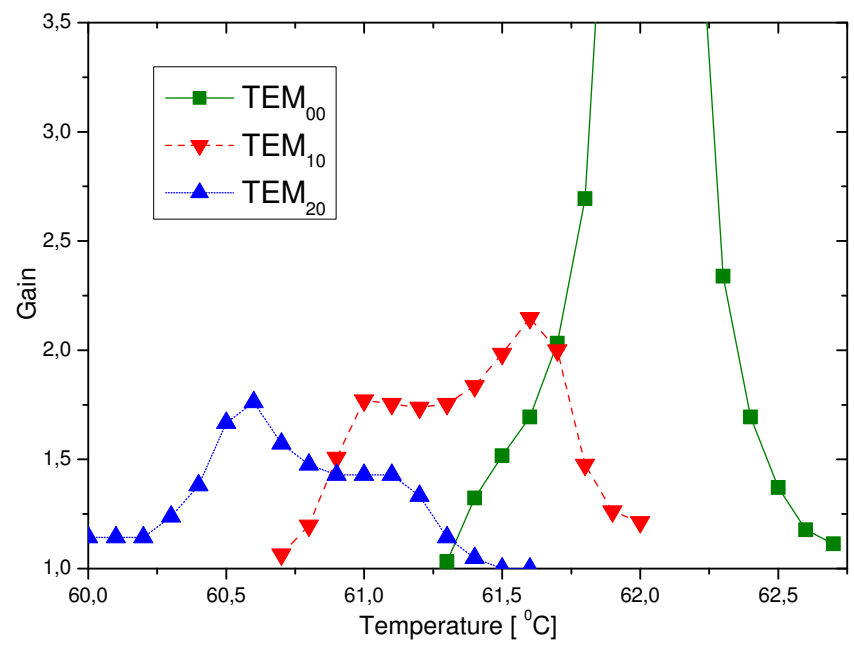

FIC. 2 Classical gain factor as a function of the crystal temperature for OPA operation with a $\mathrm{TEM}_{00}$, a $\mathrm{TEM}_{10}$ and a $\mathrm{TEM}_{20}$ signal modes. The optimal phase-matching temperature are $62.1^{\circ} \mathrm{C}, 61.6^{\circ} \mathrm{C}$ and $60.6^{\circ} \mathrm{C}$ for the $\mathrm{TEM}_{00}, \mathrm{TEM}_{10}$ and $\mathrm{TEM}_{20} \mathrm{H}-\mathrm{C}$ modes, respectively.

The measured amplification gain curves for $\mathrm{TEM}_{00}, \mathrm{TEM}_{10}$ and $\mathrm{TEM}_{20} \mathrm{H}-\mathrm{G}$ modes are presented in Figure 3. We measured a maximum amplification of 300, 23, 5 for the TEM $\mathrm{T}_{00}$, $\mathrm{TEM}_{10}$ and $\mathrm{TEM}_{20} \mathrm{H}-\mathrm{G}$ modes, respectively. The best measured de-amplification factors are $0.30,0.56$ and 0.70 for the $\mathrm{TEM}_{00}, \mathrm{TEM}_{10}$ and $\mathrm{TEM}_{20} \mathrm{H}-\mathrm{G}$ modes, respectively. On one hand, the de-amplification of 0.30 for the $\mathrm{TEM}_{00}$ indicates that the system is close to the oscillation threshold, since the theoretical value for de-amplification from a back seeded OPA at threshold is 0.25 . On the other hand, the threshold is far from being reached for the $\mathrm{TEM}_{10}$ and $\mathrm{TEM}_{20}$ modes cases. The reason why the de-amplification curves are flat and do not reach the theoretical value of 0.25 is due to mode-mismatch between the pump and seed which induces phase noises and losses.

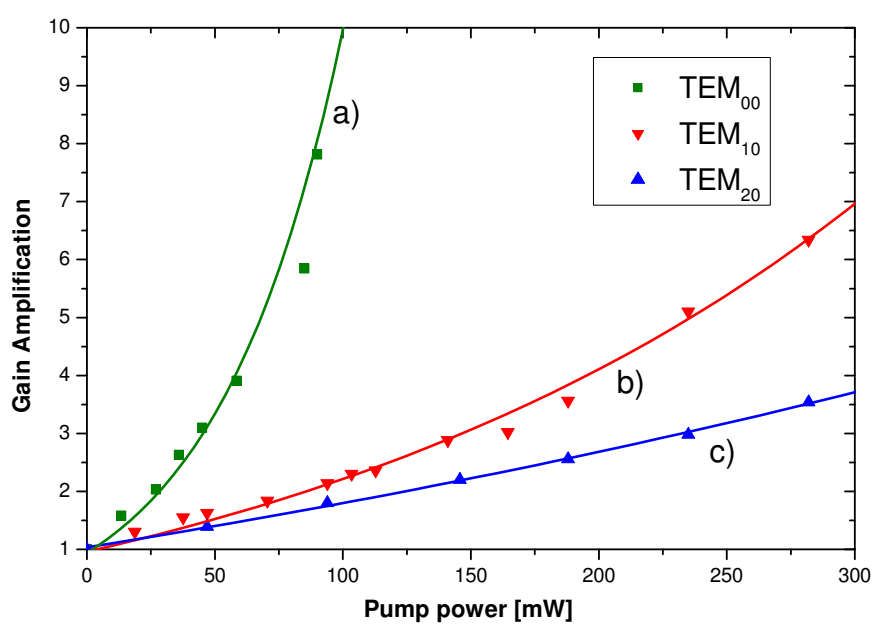

FIG. 3 Experimental classical amplification gain curves for the OPA. a) $\mathrm{TEM}_{00}$, b) $\mathrm{TEM}_{10}$ and c) $\mathrm{TEM}_{20} \mathrm{H}-\mathrm{C}$ modes. The solid lines are exponential fits.

The oscillation threshold is measured to be $260 \mathrm{~mW}$ of pump power when the OPA is resonant for the $\mathrm{TEM}_{00}$ signal mode. However, the threshold for higher order modes cannot be accessed experimentally because the system starts to oscillate on the $\mathrm{TEM}_{00}$ as soon as the pump power reaches approximately $350 \mathrm{~mW}$, even when the crystal temperatures are optimised for an operation on the TEM 10 and TEM 20 modes. Nevertheless, we can use the gain curves obtained experimentally for the $\mathrm{TEM}_{10}$ and $\mathrm{TEM}_{20}$ modes with a $\mathrm{TEM}_{00}$ pump in order to estimate the threshold for the $\mathrm{TEM}_{10}$ and TEM 20 modes. A linear fit of the first couple of data points gives the relative gain slopes between the $\mathrm{TEM}_{10}, \mathrm{TEM}_{20}$ and $\mathrm{TEM}_{00}$ modes, yielding an estimate of the relative threshold. We find the thresholds for the $\mathrm{TEM}_{10}$ and $\mathrm{TEM}_{20}$ OPA operation regimes to be approximately $1000 \mathrm{~mW}$ and $1600 \mathrm{~mW}$, respectively. These values can be compared with a theoretical calculation of the thresholds introduced in Section 2, taking into account the imperfect spatial overlap between the infrared mode resonant in the cavity and the $\mathrm{TEM}_{00}$ pump mode and the lower local intensity for higher order modes. This comparison is presented in Table 1, showing a very good agreement between theory and the experimental measurements.

We have estimated the threshold for each operation of the OPA on higher order modes, which will allow a calculation of the maximum amount of squeezing that can be generated by the system in the next section.

\begin{tabular}{|l|c|c|c|}
\hline & TEM $_{00}$ & TEM $_{10}$ & TEM $_{20}$ \\
\hline Experimental & 1 & $3.9 \pm 0.5$ & $6.2 \pm 0.8$ \\
Theoretical & 1 & 4 & 7 \\
\hline
\end{tabular}

TABLE 1 Threshold comparison between experimental results and theory in the thin crystal approximation. The threshold for the different modes is normalised with respect to the $\mathrm{TEM}_{00}$ threshold power. 


\section{TEM 00, TEM 10 and TEM 20 SQUEEZIN G}
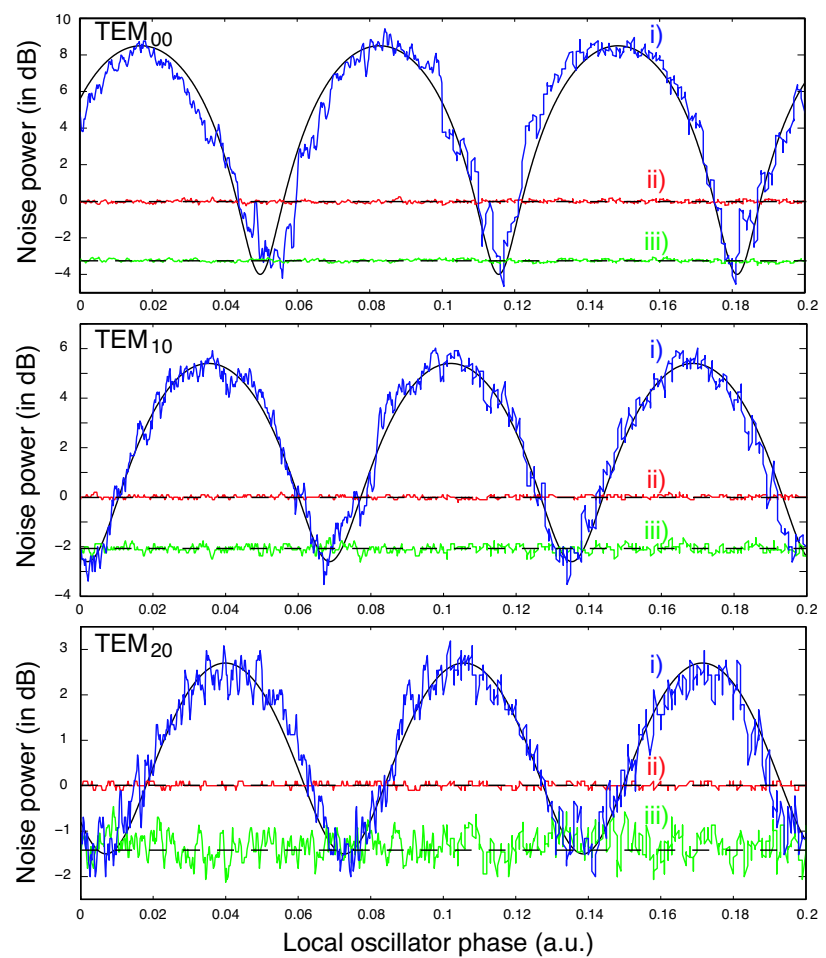

FIG. 4 Experimental squeezing traces on the a) $\mathrm{TEM}_{00}$, b) $\mathrm{TEM}_{10}$ and c) $\mathrm{TEM}_{20}$ modes, recorded by homodyne detection. i) Scan of the relative phase between the $L 0$ and the squeezed beam. ii) Quantum noise limit. iii) Phase of the LO locked to the squeezed amplitude quadrature.

The experimental squeezing curves in the $\mathrm{TEM}_{00}, \mathrm{TEM}_{10}$ and $\mathrm{TEM}_{20}$ modes are shown in Figure 4. The squeezing spectra are recorded on a spectrum analyser with a resolution bandwidth of $300 \mathrm{kHz}$ and video bandwidth of $300 \mathrm{~Hz}$ at a detection frequency of $4.5 \mathrm{MHz}$. All traces are normalised to the quantum noise level (QNL). The QNL given by trace ii) is measured by blocking the squeezed beam before the homodyne detector. Trace i) is obtained by scanning the phase of the LO, and trace iii) by locking the LO phase to the squeezed amplitude quadrature. The smooth line is the theoretical fit of the noise variance assuming the actually experimental parameters. We measured $-4.0 \pm 0.2 \mathrm{~dB}$ of squeezing and $+8.5 \pm$ $0.5 \mathrm{~dB}$ of anti-squeezing for the $\mathrm{TEM}_{00}$ mode, $-2.6 \pm 0.2 \mathrm{~dB}$ of squeezing and $+5.4 \pm 0.4 \mathrm{~dB}$ of anti-squeezing for the $\mathrm{TEM}_{10}$ mode, and $-1.5 \pm 0.3 \mathrm{~dB}$ of squeezing and $+2.7 \pm 0.4 \mathrm{~dB}$ of anti-squeezing for the $\mathrm{TEM}_{20}$ mode. To our knowledge, this is the first demonstration of higher order transverse mode squeezing using an OPA. These values have been corrected for electrical noise, which is $9.1 \pm 0.1 \mathrm{~dB}$ below the QNL, and is mostly due to the amplifiers in the photo-detectors.

In order to perform these measurements, we used the following pump powers which were maximising the amount of squeezing: $100 \mathrm{~mW}$ for the $\mathrm{TEM}_{00}$ mode and $300 \mathrm{~mW}$ for $\mathrm{TEM}_{10}$ and $\mathrm{TEM}_{20}$ modes. We were unable to pump the OPA with higher power, as the system was starting to oscillate on the $\mathrm{TEM}_{00}$ mode at $300 \mathrm{~mW}$, even when the cavity was locked to another mode, as discussed in the previous section.
We can calculate an upper limit for the measurable noise variance of the amplitude $V^{-}$and phase $V^{+}$quadratures at zero frequency, using the following simple analytical expression [28]:

$$
V^{ \pm}(0)=1 \pm \eta_{e s c} \frac{4 \sqrt{P / P_{t h r}}}{\left(1 \mp \sqrt{P / P_{t h r}}\right)^{2}},
$$

where $P$ and $P_{t h r}$ correspond to pump and threshold power, respectively $; \eta_{e s c}=T /(T+L)$ is the escape efficiency, where $L$ is the intra-cavity loss and $T$ is the transmittance of the output-coupler. The estimated intra-cavity losses for our OPA is approximately $L=0.0043$, where we consider absorption in the material $(0.1 \% / \mathrm{cm})$ and scattering at the mirror and crystal. This gives a cavity escape efficiency of approximately $\eta_{c a v}$ $=0.89$. The calculated squeezing and anti-squeezing for the different modes, using Eq.(5) are shown in Table 2c.

From Figure 4, we can see that the noise spectrum from the OPA is far away from the minimum uncertainty state predicted for a lossless OPA. We will in the following estimate and characterise the losses in our experiment.

The total detection efficiency of our experiment, is given by: $\eta_{\text {total }}=\eta_{\text {cav }} \eta_{\text {prop }} \eta_{\text {det }} \eta_{\text {hd }}$, where $\eta_{\text {prop }}=0.97 \pm 0.02$ is the propagation efficiency, $\eta_{\text {det }}=0.93 \pm 0.05$ is the photodetector (Epitaxx ETX500) efficiency. $\eta_{h d}$ is the homodyne detection efficiency. By carefully optimising the homodyne detection efficiency we measured $\eta_{h d}\left(T E M_{00}\right)=0.98 \pm 0.02$, $\eta_{h d}\left(T E M_{10}\right)=0.95 \pm 0.02$ and $\eta_{h d}\left(T E M_{20}\right)=0.91 \pm 0.02$ for the different modes. The mode dependence is firstly due to the larger spatial extension of higher order modes, which are more apertured by the optics. Moreover, the fringe visibility drops for higher order modes because of the additional transverse degree of freedom compared to the $\mathrm{TEM}_{00}$ case. Finally, a small mode mismatch has more dramatic effects on the fringe visibility for a complex intensity distribution. The total estimated detection efficiencies for our experiment are therefore $\eta_{\text {total }}=0.79 \pm 0.04, \eta_{\text {total }}=0.76 \pm 0.04$ and $\eta_{\text {total }}=0.73 \pm 0.04$ for the $\mathrm{TEM}_{00}, \mathrm{TEM}_{10}$ and $\mathrm{TEM}_{20}$, respectively. From these efficiencies can we infer squeezing and antisqueezing values, see Table 2 . In order to compare these efficiencies, we can calculate the total theoretical detection efficiency from the squeezing spectrum. The theoretical detection efficiency for a lossy OPA is given by:

$$
\eta=\frac{V_{S Q L}+V_{A S Q L}-1-V_{S Q L} V_{A S Q L}}{V_{S Q L}+V_{A S Q L}-2},
$$

where $V_{S Q L}$ is the measured squeezing, $V_{A S Q L}$ is the measured anti-squeezing and $\eta$ is the total efficiency of the experiment. The calculated efficiencies can be seen in Table 3 .

\begin{tabular}{|l|c|c|c|c|c|c|}
\hline & \multicolumn{2}{|c|}{ TEM $_{00}$} & \multicolumn{2}{c|}{ TEM $_{10}$} & \multicolumn{2}{c|}{ TEM $_{20}$} \\
\hline a) Corrected & -4.0 & +8.5 & -2.6 & +5.4 & -1.5 & +2.7 \\
b) Inferred & -5.1 & +9.0 & -3.2 & +5.9 & -1.9 & +3.1 \\
c) Calculated & -7.6 & +11.0 & -6.8 & +9.1 & -5.4 & +6.5 \\
\hline
\end{tabular}

TABLE 2 TEM $_{n 0}$ mode squeezing and anti-squeezing a) Corrected for electronic noise. b) Inferred by taking detection and propagation losses into account. c) Calculated taking the cavity escape efficiency and relative pump power into account.

The discrepancy between the calculated and the estimated efficiencies, suggests that additional losses are present in the 
system. A common extra possible loss factor is given by green induced infra-red absorption (GRIIRA) [29]. But according to Furakawa et al. no effect of GRIIRA should be seen in our setup as we have chosen a $7 \% \mathrm{MgO}$ doped $\mathrm{LiNbO}_{3}$ crystal. Nevertheless, we find that the losses seem to be induced by the pump and are dependent on the H-G mode intensity distribution and are therefore more important in an OPA pumped with a non-optimal pump. Eq.(3) and Eq.(4) show that the mode-overlap between the $\mathrm{TEM}_{00}$ pump and the $\mathrm{TEM}_{10}$ and $\mathrm{TEM}_{20}$ seed are only 0.58 and 0.47 , respectively. Therefore more losses are induced and degrade the measurable squeezing for the higher order H-G modes. This is also what we find from the theoretical calculated efficiency, Eq. (6). The comparison suggest that we have a decrease in the cavity escape efficiency due to absorption in the crystal to $\eta_{c a v, 00}=$ $0.76 \pm 0.02, \eta_{c a v, 10}=0.62 \pm 0.02$ and $\eta_{c a v, 20}=0.53 \pm 0.02$ for the $\mathrm{TEM}_{00}, \mathrm{TEM}_{10}$ and $\mathrm{TEM}_{20}$, respectively. This indicates that the absorption is getting larger for higher order $\mathrm{H}-\mathrm{G}$ modes due to the decrease in mode-overlap between the (non-optimal) pump mode and the higher order H-G signal mode resonant in the OPA, which is in good agreement with the simple mode-overlap model.

\begin{tabular}{|c|c|c|c|}
\hline & TEM $_{00}$ & TEM $_{10}$ & TEM $_{20}$ \\
\hline Est. eff. & $0.79 \pm 0.04$ & $0.76 \pm 0.04$ & $0.73 \pm 0.04$ \\
Cal. eff. & $0.67 \pm 0.03$ & $0.53 \pm 0.03$ & $0.40 \pm 0.03$ \\
\hline
\end{tabular}

TABLE 3 Comparison between estimated and calculated detection efficiency in our system.

Losses are at this point limiting the amount of measurable squeezing in the higher order H-G modes. However, we believe that there is a potential for further improvement. A next experimental proposal would be to generate optimal pump profiles in order to pump the OPA more efficiently. As previously discussed, a way of achieving perfect mode-matching between the $\mathrm{TEM}_{10}$ seed and the pump is by creating a "multimode" pump beam consisting of a mixture of $\mathrm{TEM}_{00}$ and $\mathrm{TEM}_{20}$. For the TEM 20 mode, the pump mode should be a mixture of TEM $00, \mathrm{TEM}_{20}$ and $\mathrm{TEM}_{40}$ modes. This multi-mode pump mode can be synthesise using a spatial light modulator or by using a series of mode transferring cavities. The later method for synthesise the multi-mode is experimental very challenging.

\section{C O N CLUSION}

In this contribution we demonstrate the generation of squeezed light in the $\mathrm{TEM}_{00}, \mathrm{TEM}_{10}$ and $\mathrm{TEM}_{20}$ modes. To our knowledge this is the first demonstration of higher order transverse mode squeezing using an OPA. Losses in the material limit us presently to noise suppressions of $4 \mathrm{~dB},-2.6 \mathrm{~dB}$ and $-1.5 \mathrm{~dB}$. However, we can infer about $-7 \mathrm{~dB}$ and $-5 \mathrm{~dB}$ of noise suppression inside the OPA. This is similar to the degree of squeezing observed in conventional CW quantum optic experiments and we believe that future improvements of our setup will allow us to increase the observed amount of squeezing. We propose a method to maximise the measurable squeezing of the present system using a spatial light modulator to synthesise the multi-mode pump mode to generate the optimal pump profiles for pumping the OPA more efficiently and to minimise the nonlinear intra-cavity losses.

Using the transverse spatial properties of laser beams, we are now able to produce all the required tools for higher order continuous laser quantum optics experiments. The key components are the ability to generate the H-G modes selectively with high efficiency and the availability of simple and fully efficient modulation and detection techniques. The way for parallel quantum information processing with continuous variables in the transverse plane of a laser beam using the basis of H-G modes is now open. In order to test parallel quantum information protocols, we plan for future experiments to produce spatial entanglement using two higher H-G mode squeezers. Other types of experiments that could follow are dense coding of spatial information, teleportation of spatial information and spatial holography.

\section{Acknowledge me nt}

We would like to thank Preben Buchhave, Jiri Janousek and Magnus Hsu for many useful discussions. This work was supported by the Australian Research Council Centre of Excellence scheme. ML is supported by the Danish Technical Research Council (STVF Project No. 26-03-0304).

\section{References}

[1] L. Lugiato, A. Gatti, and E. Brambilla, "Quantum imaging", Journal of Optics B: Quantum and Semiclassical Optics 4 S176-S183 (2002).

[2] M. Kolobov, "The spatial behavior of nonclassical light", Reviews of Modern Physics 71 1539-1589 (1999).

[3] S. Gigan, L. Lopez, V. Delaubert, N. Treps, C. Fabre, and A. Maitre, "Continuous-wave phase-sensitive parametric image amplification", Journal of Modern Optics 53 809-820 (2006).

[4] N. Treps, U. Andersen, B. Buchler, P. K. Lam, A. Maitre, H.-A. Bachor, and C. Fabre, "Surpassing the standard quantum limit for optical imaging using non-classical multimode light.", Phys. Rev. Lett. 88203601 (2002).

[5] V. Delaubert, N. Treps, C. Harb, P. K. Lam, and B. H.-A., "Quantum measurements of spatial conjugate variables: Displacement and tilt of a Gaussian beam", Opt. Lett. Doc. ID66257 (2006).

[6] I. Sokolov, M. Kolobov, A. Gatti, and L. Lugiato, "Quantum holographic teleportation", Optics Communications 193 175-180 (2001).

[7] M. I. Kolobov and L. A. Lugiato, "Noiseless amplification of optical images", Physical Review A. Atomic, Molecular, and Optical Physics 52 4930-4940 (1995).

[8] C. Caves and P. Drummond, "Quantum limits on bosonic communication rates.", Rev. Mod. Phys. 66481 (1994).

[9] A. Zeilinger, "Quantum entangled bits step closer to IT.", Science. 289405 (2000).

[10] S. Oemrawsingh, A. Aiello, E. Eliel, G. Nienhuis, and J. Woerdman, "How to observe high- dimensional two-photon entanglement with only two detectors", Rev. Rev. Lett. 92 217901-1 (2004). 
[11] N. Langford, R. Dalton, M. Harvey, J. OBrien, G. Pryde, A. Gilchrist, S. Bartlett, and A. White, "Measuring entangled qutrits and their use for quantum bit commitment", Phys. Rev. Lett. 93 053601-1 (2004).

[12] S. Barnett and R. Zambrini, "Resolution in rotation measurements", J. Mod. Opt. 53613 (2005).

[13] A. Siegman, 'Lasers' (University Science, Mill Valley California, 1986).

[14] H. Kogelnik, "Coupling and conversion coefficients for optical modes", Proceedings of the Symposium on Quasi-Optics (1964).

[15] E. Morrison, B. Meers, D. Robertson, and H. Ward, "Automatic alignment of optical interferometers", Applied Optics 33 5041-5049 (1994).

[16] H.-A. Bachor, V. Delaubert, C. Harb, M. Hsu, P. Lam, C. Fabre, and N. Treps, "Spatial quantum effects with continuous-wave laser beams.", Journal of Modern Optics 53597 (2006).

[17] L. Wu, H. Kimble, J. Hall, and H. Wu, "Generation of squeezed states by parametric down conversion.", Phys. Rev. Lett. 572520 (1986).

[18] P. Lam, T. Ralph, B. Buchler, D. McClelland, H.-A. Bachor, and J. Gao, "Optimization and transfer of vacuum squeezing from an optical parametric oscillator", Journal of Optics B: Quantum and Semiclassical Optics 1 469-474 (1999).

[19] K. McKenzie, N. Grosse, W. Bowen, S. Whitcomb, M. Gray, D. McClelland, and P. Lam, "Squeezing in the audio gravitational-wave detection band", Phys. Rev. Lett. 93161105 (2004).

[20] R. Boyd, Nonlinear Optics (Academic Press, San Diego, 1992).
[21] A. Vaziri, G. Weihs, and A. Zeilinger, "Superpositions of the orbital angular momentum for applications in quantum experiments", Journal of Optics B: Quantum and Semiclassical Optics 4 S47-S51 (2002).

[22] M. Lassen, P. Tidemand-Lichtenberg, and P. Buchhave, "Simultaneous measurement of patterns in the signal and idler near and far fields from a confocal optical parametrical oscillator", Physical Review A 72 1-9 (2005).

[23] U. Schwarz, M. Bandres, and J. Gutierrez-Vega, "Observation of Ince-Gaussian modes in stable resonators", Optics Letters 29 18701872 (2004).

[24] C. Schwob, P. Cohadon, C. Fabre, M. Marte, H. Ritsch, A. Gatti, and L. Lugiato, "Transverse effects and mode couplings in OPOS", Applied Physics B Lasers and Optics 66 685-699 (1998).

[25] R. Drever, J. Hall, F. Kowalski, J. Hough, G. Ford, A. Munley, and H. Ward, "Laser phase and frequency stabilization using an optical resonator", Applied Physics B: Photophysics and Laser Chemistry B31 97-105 (1983).

[26] Diabolo laser from Innolight, www.innolight.de.

[27] V. Delaubert, M. Lassen, N. Treps, C. Harb, P. K. Lam, and B. H.A., "Selective generation of spatial modes in a single pass second harmonic generation", To be submitted (2006).

[28] H.-A. Bachor and T. C. Ralph, A Guide to Experiments in Quantum Optics, 2nd edition (Wiley, 2004).

[29] Y. Furukawa, A. Alexandrovski, R. Route, M. Fejer, and G. Foulon, "Green-induced infrared absorption in MgO doped $\mathrm{LiNbO}_{3}$ ", Appl. Phys. Lett. 781970 (2001). 